\title{
A Proposed Electronic Health Record Content Structure Based on Clinical Organizations Survey
}

\author{
Dr. Ayman E. Khedr, Fahad Kamal Alsheref \\ Information Systems Department, Faculty of Computer Science and Information, Helwan University, \\ Cairo, Egypt \\ aymankhedr@helwan.org.eg \\ e.fahad.kamal@gmail.com
}

\begin{abstract}
Computer systems and communication technologies made a strong and influential presence in the different fields of medicine. The cornerstone of a functional medical information system is the Electronic Health Records (EHR) management system. Several electronic health records systems were implemented in different states with different clinical data structures that prevent data exchange between systems even in the same state. This leads to the important barrier in implementing EHR system which is the lack of standards of EHR clinical data structure.

In this paper we made a survey on several in international and Egyptian medical organization for implementing electronic health record systems for finding the best electronic health record clinical data structure that contains all patient's medical data. We proposed an electronic health record system with a standard clinical data structure based on the international and Egyptian medical organization survey and with avoiding the limitations in the other electronic health record that exists in the survey.
\end{abstract}

\section{Indexing terms/Keywords}

Keywords: Electronic Health Record; Standards of Medical Data; Unstructured Medical Data; Health Information Technology.

\section{Academic Discipline and Sub-Disciplines}

The main discipline is medical information system, Health information systems and Electronic health record.

\section{Council for Innovative Research}

Peer Review Research Publishing System

Journal: INTERNATIONAL JOURNAL OF COMPUTERS \& TECHNOLOGY

Vol.13, No.12

www.ijctonline.com, editorijctonline@gmail.com 


\section{Introduction}

Health Information Technology (HIT) allows comprehensive management of medical knowledge and its secure exchange among health care consumers and providers. Broad uses of HIT will: help to eliminate the manual tasks of extracting data from charts or filling out specialized datasheets, help to derive data directly from the electronic record, making research-data collection by product of routine clinical record keeping, help to Move from paper-based health care system to secure electronic medical records which will save lives and reduce health care costs and help in Early detection of infectious disease by Advanced data collection, fusion and processing techniques which would be at the forefront in spotting the emergence of new diseases, and crucial to tracking the spread of known diseases [1].

Electronic Health Record (EHR) is a collection of all patient medical information from multiple resources including all the components of EHR accessible from any location by any provider caring for the patient. The components of EHR can be [1] [2]: patient demographics. medical history, examination and progress reports of health and illnesses, medicine and allergy lists, scheduling, retrieval, and archiving of laboratory tests, graphic image display, medication ordering including patient safety functions, clinical practice guidelines, appointment scheduling and claims and payment processing [3].

The application of Electronic Medical Record (EMR) systems helps to improve the quality level of patient care and decrease medical errors. It also may help in [3]: integrated decision support and guidance, security, privacy, and confidentiality standards for clinical terminology, integrating the patient record with other information resources in the organization and financial benefits.

The rest of this paper is organized as follows. Section 2 provides the background of Electronic Health record, implementation models and barriers. Section 3 provides survey of electronic health record implementation attempts in several countries like Finland, France and Denmark. Section 4 provides survey of electronic health record implementation attempts in Egypt. Section 5 provides the proposed electronic health record components with their structural contents. Finally, Section 6 contains the conclusion and future work.

\section{Electronic Health Record}

The Electronic Health Record (EHR) is defined as longitudinal electronic record of patients' health information generated by one or more encounters in any care delivery setting. This information includes, but is not limited to, patient demographics, progress notes, examinations details like symptoms and findings, medications, vital signs, past medical history, immunizations, laboratory data, and radiology reports. The EHR automates and streamlines the clinician's workflow. The EHR has the ability to generate a complete record of a clinical patient encounter as well as supporting other care directly or indirectly related activities via interface including evidence-based decision support, quality management and outcomes reporting. The EHR means a repository of patient data in a digital form stored and exchanged securely and accessible by multiple authorized users [2][3].

\section{Electronic Health Record Implementation models}

There are many EHR architectural models that can be used all over the world. The most two popular EHR models are:

\section{a. Central Repository Model}

The center of EHR model will be the repository, which will be fed by the existing applications in different care locations such as hospitals, clinics, and family physician practices. The feed from these applications will be messaging based on the pre-agreed standards. The messaging needs to be based well-defined standards, for example the HL7. Reference Information Model (RIM) for which XML could be used as the recommended Implementation Technology Specification (ITS) [4].

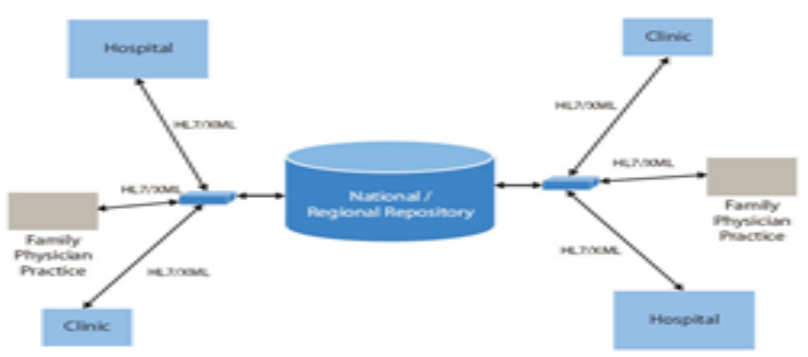

Fig1: EHR Central Repository Model [4] 
The event-driven messages that need to be sent and stored in the repository will essentially be event-based summaries as shown in figure (2). The event-based summaries stored in the repository can be queried and retrieved by different clinicians who are treating the patients in different scenarios and by different clinical settings. The retrieval and access of data from the repository is subject to establishing that the clinicians are legitimately accessing the data for treating only those patients who are in their care. The retrieval is done through messaging which can be done either through synchronous or asynchronous messages depending on the urgency, complexity, and importance of the data that is being retrieved [4].

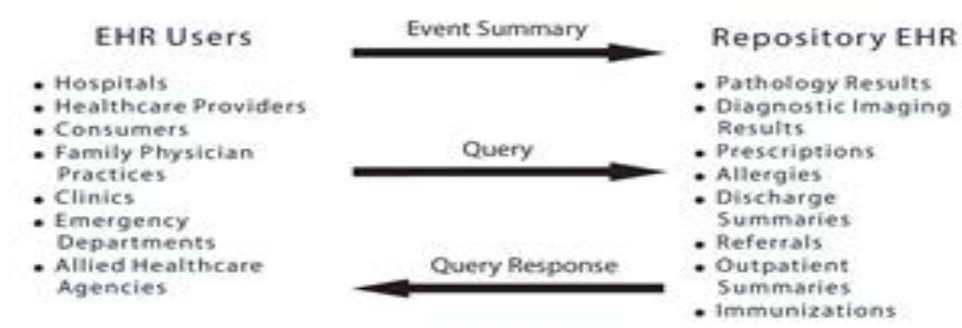

Fig2: EHR Message Events [4]

\section{b. Managed Services Model}

The managed services model is based on hosting applications for different care providers and care settings in a data center by a consortium, which may consist of group of infrastructure providers, system integrators, and application providers. The hosted applications can be used to provide an effective EHR by building a common repository using a shared database or by providing a common user interface to all hosted applications and extracting data from these systems using a portal whose authentication and authorization mechanism can also be controlled at the data center level as depicted in figure 3 [4].

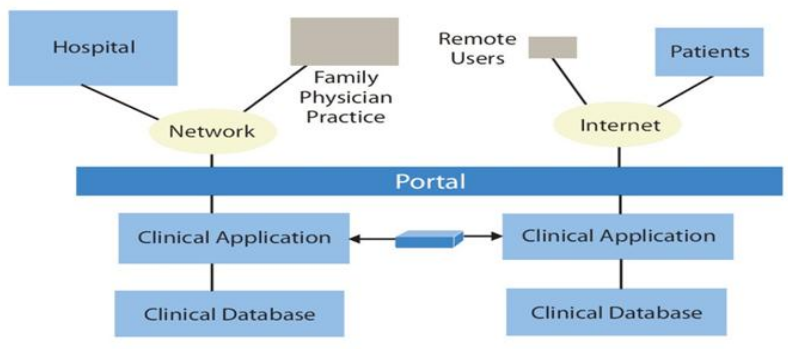

Fig3: Shared Services Model [4]

\section{Electronic Health Record implementation barriers}

Implementation of EHR faces different barriers, but these barriers vary from one environment to another. Hereafter, the main focus will be on the general barriers that exist in most of EHR implementation attempts, these barriers are:

\section{a. Financial Barriers}

Financial barriers are divided into the following points:

- High Costs: These costs are divided into two main parts, initial cost and ongoing cost .The initial cost is high because it includes the cost of EHR hardware and software equipment, staff training, and interface installation. Ongoing costs are associated with the hardware update, software upgrades and so on [5].

- Under-developed business case: This barrier is raised because of the following [5]:

- Uncertainty of EHR returns on investment,

- Financial benefits are only achieved on the long run,

- The main objective and benefits of EHR is to provide a high quality medical service for the citizens. 


\section{b. Technological Barriers}

Technological barriers are divided into the following main views [6]:

- Inadequate technical support: There is high cost in Time for obtaining technical support from the various software, hardware, networking, backups, and service vendors.

- Inadequate data exchange: Another barrier to EHR use which is the lack of adequate electronic data exchange between the EMR and other clinical data systems (such as lab, radiology, and referral systems). Having parallel electronic and paper based systems forced physicians to switch between systems, thereby slowing workflow, requiring more time to manually enter data from external systems, and increasing physicians' resistance to EHR use.

- Security and privacy: Privacy, confidentiality and security of electronic medical records and internetbased communication are great barriers for EHR system adoption, because of the importance of the clinical information of the patients that only accessible by authorized clinical professionals .also there are important techniques should be used like (electronic signature, encryption techniques, etc.) to ensure the confidentially of clinical data and protection of the clinical data from unauthorized persons.

\section{c. Organizational Change Barriers}

This category contains many points; these points are as following [7]:

- Design of and alignment with workflow and office integration: 54.2 percent out of the 5000 respondents reported that they are worried about slower workflow and low productivity according to the American Academy of Family Physicians survey results (American Academy of Family Physicians 2004). An EHR that does not integrate smoothly into the physicians' workflow and does not allow for variations in style can negatively affect productivity and financial return (American Academy of Family Physicians 2004). Another recent survey of IT vendor organizations reported that workflow design, system integration, and network support were areas in which health care organizations have staffing needs (American Academy of Family Physicians 2004). There are some concerns regarding the initial disruption of office workflow associated with the introduction of new IT (Information Technology) systems and how to align these systems into the practitioner's workflow.

- Migration from paper-based systems: Moving to a paperless system may create an initial disruption in some financial, clinical and organizational processes. Having initially paperless and paper systems concurrently represents an additional concern.

- Staff training: Staff training to use a new tool may temporarily reduce productivity. Also, there are questions about the types of training and skills needed to adopt IT systems for health care quality.

\section{d. Lack of standards}

The standards rule is a key element of a national push to get doctors and hospitals across the country to adopt electronic health records. If two different systems use a common standard, they can communicate with each other, or "interoperate." When systems are interoperable, information from one system can be sent into another with little or no translation. It is somewhat analogous to being able to write a document in Microsoft Word on a Windows PC and have it read perfectly by someone using an Apple computer system [8].

A health care system that uses electronic health records sporadically, and also has no common software standards, can be compared to a tower of Babel. That's largely the situation today in the United States. As things stand, many patient records are still on paper, and it's difficult to get comprehensive data on a lot of patients from them (a process known as "pulling charts"). Compiling that data would also be cumber some. Even dealing with on patient's records can be difficult when a patient leaves one hospital for another. It's time consuming and expensive to copy the records or otherwise transmit them to the next hospital through the mail, by fax, or by hand. As a result, vital patient records aren't always shared. Even where electronic health records are in use today, providers may not readily share data because of different system standards or in some cases, because no standards or only partial standards are in place. This means that records in one system are not easily transferred to another [8].

\section{e. Clinical Data store shape in EHR systems}

Generally speaking, there are two main types of data store shapes: structured data and unstructured data. Unstructured data consists of two basic categories [9]: 
- $\quad$ Bitmap Objects: Inherently non-language based, such as X-rays, radiology, video or audio files.

- Textual Objects: Based on a written or printed language, such as clinical reports, nursery notes and examination sheets.

Using unstructured data for storing clinical data has the following limitations [9]:

Even if unstructured data is in a well-defined format such as a template, the data is still not consumable from a semantic level without a compatible interface or application. Even with a compatible technology, it cannot be necessarily gained insight into the context of the information unless it can actually be read.

\section{Electronic Health Record implementation barriers analysis}

In this section, previous barriers and adopted solutions are analysed in addition to identifying the extension of applying these solutions to solve the problem partially or totally, the adopted solutions are [10][11]:

a. Financial Barriers analysis:

Solutions for the financial barriers are working in two different stages: reducing the initial cost and reducing the cost in the long run like:

- Modular system.

- $\quad$ Time savings realized in the long run.

b. Technological Barriers analysis:

The adopted solutions for technological barriers are:

- $\quad$ Arranging comprehensive, technical and process support:

- National movement towards the adoption of EHR standards.

c. Organizational Change Barriers analysis:

The following solutions are adopted for office workflow and migration from paper system to electronic system:

- $\quad$ EHR systems increase office workflow, clinical support, and systems integration.

- $\quad$ Preparing to incorporate IT systems in daily office workflow.

- $\quad$ Office Workflow Analysis.

- $\quad$ Avoiding the use of electronic and paper based systems in parallel.

- Maximizing electronic data exchange to reduce data entry.

\section{EHR Implementations: case problems}

There are several attempts for implementing EHR in different countries; each attempt has its strength and weak points, the following sections show the attempts in Finland, France and Denmark and their shared barriers.

\section{Finland EHR Attempt}

Finland's e-health road map built on the 1996 strategy. The road sets the country's strategic objectives on ehealth. This canter on secure access to information for those involved in care regardless of time or place, together with increased citizen access to high quality health information. The focus of the strategy is the development of a national $\mathrm{E}$ Archive record repository and E-Prescribing service. The interoperability of health care information systems, in particular patient record systems, is one of the Finland's main e-health aims. It began to address the issue through legislation, national recommendations and specifications.

The basic principles of the national health care information system architecture were outlined by the ministerial committee on information society program back in March 2006. This was swiftly turned into bills and passed into law in February 2007. Two Acts of Law were passed: (a) the Act on the electronic processing of health care and social welfare client data; and (b) the Act on electronic prescriptions. Between them they provided the legal basis for archiving and distribution of electronic patient records; creating a national prescription database; establishing an electronic certification service for health care professionals, and a service for classifications, codes and terminology.

This regulatory, political, legal and policy framework, widespread use of data standards plus the overall high Information Technology (IT) skills of health professionals has set Finland on the road to achieving a fully functioning e-health architecture by 2012 [12][13].

So the achievements that the Finland government made: in the beginning of 200610 out of 21 hospital districts were able to share electronic patient information between health care organizations inside their regions, but exchange of electronic patient data on the national level has not been possible yet [13]. Core data elements include information for 
patient identification, clinical data such as diagnoses and medications, and risk data. Core data elements are located inside electronic medical documents from which they can be extracted and then "recycled" to be included in new documents, for example referrals, doctor's orders and medical certificates [13]. National e-Prescribing pilot-project was launched in 2002. The pilot-project ran from 2004-2006. In this project a prescription database was built by the National Social Security Agency, and e-signature systems and connections from health care providers and pharmacies to the database were experimented [13]. Smart Citizen ID-card is in production. This card is managed by The Population Register Centre. About 60.000 cards have been distributed and this ID number is equivalent to the Social Security Number [13]. National health portals were opened, portals for patients and a portal for professionals. Finland attempt was promising and achieved several goals of using that systems but it did not has a clear standard structure of clinical data to enable the interoperability of EHR systems in different organizations.

\section{France EHR Attempt}

In France the national authorities decided to promote the establishment of a personal health record which is called in French DMP that refers to (dossier medical personnel) for the entire population. The DMP was enshrined in a law passed in August 2004, with the provision that it be implemented within three years, i.e. by July 2007 [14]. The definition of the DMP specifications, its experimentation and its development were hindered by this unrealistic deadline, which eventually caused the project to be stopped in its initial form in May 2008 [14]. It was then recommended that it be continued under different conditions, more in tune with the needs of users (health professionals and citizens). Two other projects are nevertheless under development at national level, more or less complementary to the DMP project, although their link with it remains quite unclear [14]:

- $\quad$ The system now called physicians' web , developed by the CNAMTS, the main public health insurance fund for salaried workers, which will afford physicians with access to each patient's list of past drug prescriptions, lab tests (not their results) and procedures that have been reimbursed by the public health insurance.

- The pharmaceutical record (dossier pharmaceutique) (DP), a developed under the aegis of the national association of dispensing chemists, which provides access to the drugs delivered to each patient, whether prescribed or not.

To characterize the national Electronic Health policy in France, we could say that it consists of various initiatives that are not really nationally co-ordinate. Many actors worry about the absence of a coherent policy for health informatics. Although the DMP initiative has been the main one in recent national policies, its relationships with other initiatives have never been properly worked out. The DMP project is, corresponding to the years 2004-2008, is characterized by [14]:

- $\quad$ A highly centralized top-down approach, with excessive government intervention.

- An ambiguous definition of the goals pursued by the DMP as a tool, with no choice between professional use and personal use.

An inadequate analysis of its functions and uses, and consequently inappropriate technical features [14]:

- An under-estimated complexity and an extremely short time-frame to implement the system.

- A lack of instruments for steering the project: such as external evaluation, participation of the stakeholders (firms, professionals, patients, and general public), preliminary studies, technology assessment, on the whole, a largely insufficient governance of the project.

- A lack of tools for accompanying the change.

We are of the opinion that the project must also be reconfigured with a broader perspective on the reshaping of the health care system in France, and should be one of its key elements without being exclusive. Behind any project of this size, there must be at least a reflection, if not a vision and a clear plan, as to the changes to foster in the organization of the health system at large including the internal relations between the actors [14].

Finally, the project lacked an outside view, both in its definition; for example to identify users' needs, and to identify and take into account the organizational components and in its monitoring, that would have enabled it to evolve. Use of evaluators for this purpose, from the academic world or from private institutions, should avoid an amateurish approach, even if the intention is good, also the training for physicians on the modern tools like computers and new systems should be well arranged and try to find easier way to enter this huge amount of medical data.

\section{Denmark EHR Attempt}

EHR systems have been developed systematically in Denmark in the past 10 years, but it is still not possible to buy a standard EHR system to be used in a hospital. One of the instruments to focus the development and implementation is a national agreed IT strategy for the health services [15].

The Ministry of Health and Interior launched in May 2003 a national strategy for the health services. The new strategy was now no longer limited to specific topics or organizations (e.g., health care networks, EHR in hospitals), but included the 
use of IT in the entire health sector. The aim of the strategy was to support the order of priority for the use of IT in the health care service, i.e. [15]:

- Contribute directly to the improvement of quality, service and coherence in patient care.

- Ensure better communication between all parties in the health care service.

- Contribute to the fast and safe access of the individual citizen and/or patient to his own health record as well as to information concerning service and quality of health care.

- Be instrumental to better administration and management of the health care service.

- Ensure coordination with the political goals concerning digitization of the public sector in Denmark.

The strategy was very ambitious and included 29 initiatives in three areas [15]:

- Clinical workplaces in the health services.

- National registries and databases.

- Coherent access to information.

This strategy has the following strong points:

- Establishment of a Danish public health portal (www.sundhed.dk). The portal includes access to clinical and patient information as well as a number of services.

- Start translation of SNOMED CT and as a follow of this work the establishment of IHTSDO in Copenhagen.

- EHR coverage (beds) in Hospital increased to 50\% (not all systems are full EHR).

- National medication profile on Sundhed.dk.

- Internet based Health Care Network.

- Messages between Hospitals and Municipalities.

- Extract from HIS/EHR systems to a national repository.

- A lot of debates regarding EHR model, terminology and clinical content.

And also has the following weak points:

- The development of a Danish standard for EHR fails.

- No standards for EHR on national level by the end of 2007.

- A lot of negative publication in the news.

- Political discussion regarding the lack of EHR in the Danish Hospitals.

To summarize the case problems: the previous case problems contain shared barriers that could be summarized in the following points [14][15]:

- Lack of standards.

- Problems in using current EHR systems.

- Examination data is not available for search or analysis.

\section{Electronic Health Record implementation in Egypt}

In Egypt there was a governmental strategy to develop all the hospitals and supply them with the modern information technology programs and the EHR was one of these development goals but this was in the era of the minister Prof. Esmael Salam and this attempt is stopped after he leaved the ministry [16].

Now in the new Egyptian strategy for communications and information technology the Government of Egypt and its Ministry of Health have established several e-Health programs to bring better diagnostic and health services to a wider segment of the Egyptian society. MCIT (Ministry Information Communications Technology) has facilitated the integration of ICT (Information Communications Technology) in health services and the provision of medical education to remote or underserved areas of Egypt. The e-health initiative is inspired by pursuing equal opportunities for health services anywhere in Egypt, and expanding medical insurance to all citizens. The principle objectives of the e-Health initiative are as follows [16]:

- $\quad$ Extend better medical diagnostic services to rural areas. 
- $\quad$ Provide a training facility for the medical community.

- Acquire international consultations for special cases.

- $\quad$ Reduce the cost of health care through better patient management.

- Optimize utilization of expertise and resources.

- $\quad$ Provide an advanced medical service in emergencies.

- Create an electronic database for medical records.

The projects that the ministry achieved [16]:

- Emergency Medical Call Center and Ambulance Service: MCIT and the Ministry of Health and Population (MoHP) are cooperating to establish an emergency call center for Greater Cairo. The project aims to develop the Emergency Medical Service (EMS) system in the Greater Cairo area and improve the speed and efficiency of its services by providing the Egyptian Ambulance Services Authority with a computerized ambulance dispatch system.

- $\quad$ National Network for Citizen Health: The project provides the most recent automated systems to develop central management of treatment by the government, and direct patients to different therapeutic units, such as hospitals and specialized centres in the country. The project aims to develop quality control and performance evaluation systems to guarantee the delivery of subsidy to those for whom it is intended, and guarantee system efficiency. It also aims to develop the information systems and databases of the central department for citizen health treatment by the government, and connect all peripheral departments and hospitals through a Virtual Private Network.

- Information System Units in Governmental Hospitals: The project aims to facilitate the work of MoHP by establishing an information systems unit in 700 hospitals nationwide equipped with hi-speed internet PCs, a Web Cam and a scanner. In 2008, information systems units were established in ten hospitals as a pilot phase, to be replicated in 262 hospitals, 252 of which are public and central hospitals while 37 are insurance hospitals. The units are used to facilitate the national network for citizen health treatment, patients' registration, financial and administration operations and training Medical staff.

- National Healthcare Capacity Building Project: The chief aim of the project is to create a pool of highly qualified and competent healthcare professionals who are capable of designing and implementing health systems and programs. To achieve so, the project provides training to MOHP staff including doctors, nurses, administrative staff and technicians). The training program is divided into three categories: Basic IT Skills, biomedical Awareness and Biomedical Informatics Professional Training. The project will also establish a central unit that links hospitals and keeps medical records, establish an electronic radiology and lab analysis medical records database for patients and transfer and circulate radiation and lab analysis between hospitals and the central unit in Fum El Khalij.

- National Cancer Registry Program: The program utilizes state-of-the-art data mining technologies to extract a variety of health indicators for investigating reasons behind the spread of cancer. As an initial phase, the city of Aswan was selected to be enlisted in the program. A fully equipped data collection center was prepared to be ready to receive all patients' data to be registered using the latest data mining and recording applications. A high-end data transfer application was also developed to transfer patient's data to the National Cancer Archive.

- National Picture Archiving and Communications System (PACS) Project: The project aims to develop a centralized database PACS and radiology Information System (RIS) system in the main center (Center of Excellence $\mathrm{CoE}$ ) in Fum El-Khalij that integrates clinical images, scanned documents, and other non-textual data into patient's electronic medical records. A local database was also created in the eight hospitals hospital covered by the project. Each of the hospitals' databases will be automatically synchronized with the central database in Fum El Khalij.

We examined the following governmental hospitals that contain the required Electronic Health information systems, the information networks utilities and PACS systems:

- $\quad$ Ein Shams university hospitals

- Al qasr al eny French hospital

- National Cancer Institute (NCl)

- Nasser Institute Hospital

As well as, We examine the EHR that medical professionals working on and found they have an automated system which processes the following Functions [16]: 
- $\quad$ Store the patient demographics data

- Connect the central database with the national number database

- Finance

- Appointments

- $\quad$ Store the laboratory data

- $\quad$ Store the radiology data

- $\quad$ Store the pharmacy data

- $\quad$ Store the physician examinations data

The radiology physician examinations data are stored as picture (i.e. unstructured database). The patient demographics, Finance and Store the pharmacy data are stored as structured database. The laboratory data are stored as semi-structured database. This case of EHR implementation has some problems and limitations which are:

- $\quad$ There is no standard of EHR contents.

- $\quad$ There is no nursing information system: all nursing information is stored in documents of papers which means it is not available when physician needs it and will cost more time to get this data, also this data can't be used for searching or analysis and if there any accident like fire or others that means this data will be lost.

- The physician does not work directly with EHR systems: Specific employees who are responsible for entering data and manage it, physicians don't deal with the system directly but if they want to get specific data they ask the responsible employees to get the data.

- $\quad$ If we force the physicians to enter the examination data we will face the behavioural and attitudinal problems that we mentioned before, and this will costs the physician time because that will increase the time for each patients visit and will decrease the number of patients' that he could see.

For private hospitals each hospital has its information systems, we examined the following private hospitals:

- International Peace Hospital

- Peace Hospital

- Al Ganzori Hospital

We found an electronic health record system and it contains:

- $\quad$ Each hospital has its EHR system with different structure.

- $\quad$ There is not data exchange between the EHR systems because the lack of standards.

- Patient demographics: they store demographics data as a database (tables, attributes, etc).

- $\quad$ Financial: they store financial and payment reports for each patient data as a database (e.g. tables, attributes)

- $\quad$ Appointments: they store appointments data as a database (tables, attributes, etc.).

- Laboratory: devices give digital data but without connected it with patient document.

- Radiology: devices give digital data but without connected it with patient document.

- Pharmacy: they store pharmacy data as a database (tables, attributes, etc.).

- Physician examinations: Paper based only

This electronic health record system has the following limitations:

- $\quad$ There is no connection with the governmental Electronic Health record system.

- $\quad$ There is no system for nursing; So all vital signs like pressure and heart pulse is stored in paper which means it is not easy to find the required data for specific patients and also it costs time if the physician want to get this data, but if this data is stored in the digital format and in structured database will make available as soon as physician want it.

- $\quad$ There is no connection between patients database and lab system; That means in the lab system you to store the patient data again to tell the lab system that this result is belong to this patient, so there is a redundant data because you to store patients data twice once in patients database and the other in the lab database. 
- $\quad$ There is no system for radiology; Radiology images are stored inside the memory in the digital devices but it's not stored as a data base in other words there is no connection between patients' database and radiology devices.

- $\quad$ There is no system for storing physician examinations or diagnoses; Examinations sheets which written by physicians are no stored inside the system but it still in paper form which also costs time if the physician want to get this data.

- $\quad$ Core data like physician examination is not process able; Because it's not stored in the database and if they make system to stored will stored as image and by employees ,but we want to make physician work directly with system and make examinations and diagnoses available when it is wanted and make process able (search , update statistics ....etc.).

- $\quad$ A lot of document still not stored in database These documents are stored in paper format like:

- Radiology images

- Nursing sheets

- Examinations

- Diagnoses

\section{The Proposed Electronic Health record structural contents} categories:

After the survey that had been made in section 4 and section 5 we categorized the clinical data in the following

- Patient demographics

- Patient History

- Examination (Physical)

- Laboratory results

- Progress reports

- Allergy lists

- Immunization status

- Medication (Physician Investigation)

- appointment scheduling

Each component is described with its detailed contents in the following subtitles:

\section{Patient demographics}

Patient demographics form the core of the data for any medical organization. They allow for the identification of a patient and his categorization into categories for the purpose of statistical analysis.

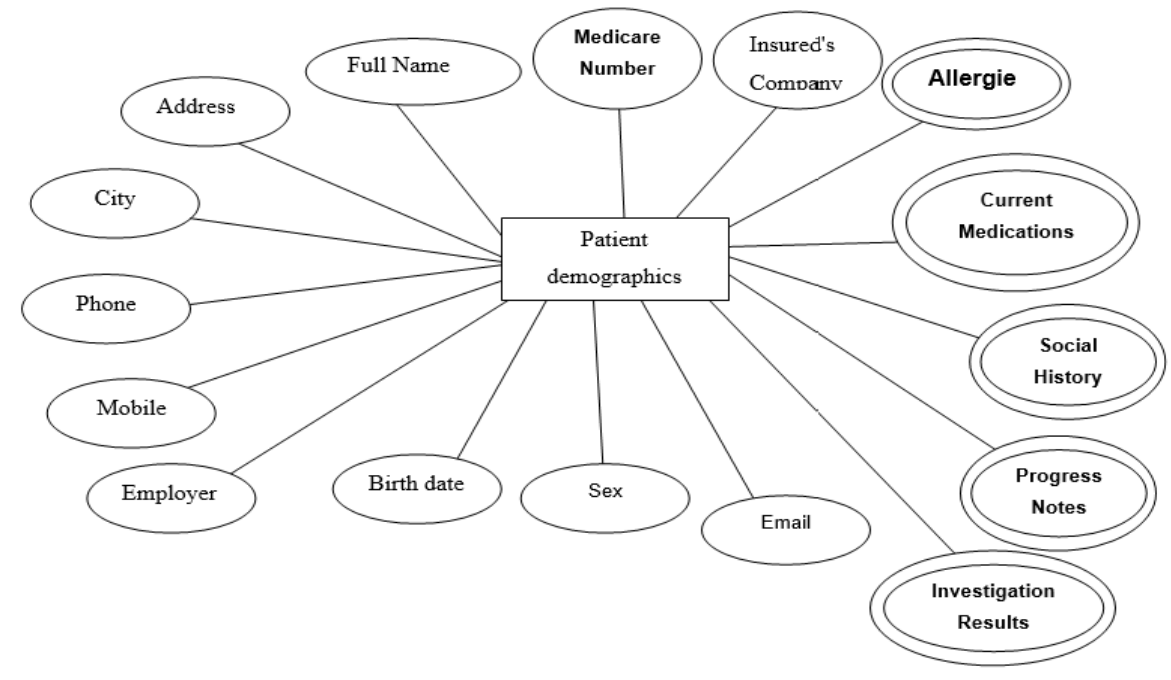

Fig 4: Patient demographics Structure 


\section{Patient History}

The patient medical history is information gained by a physician by asking specific questions, with the aim of obtaining information useful in formulating a diagnosis and providing medical care to the patient. The medically relevant complaints reported by the patient or others familiar with the patient are referred to as symptoms, in contrast with clinical signs, which are ascertained by direct examination on the part of medical personnel.

\section{Examination (Physical)}

A physical examination is a routine test that physician performs to check patient's overall health. It is also known as a wellness check. Performing an accurate physical assessment and being able to differentiate normal from abnormal findings is one of the most important roles for today's health care practitioner.

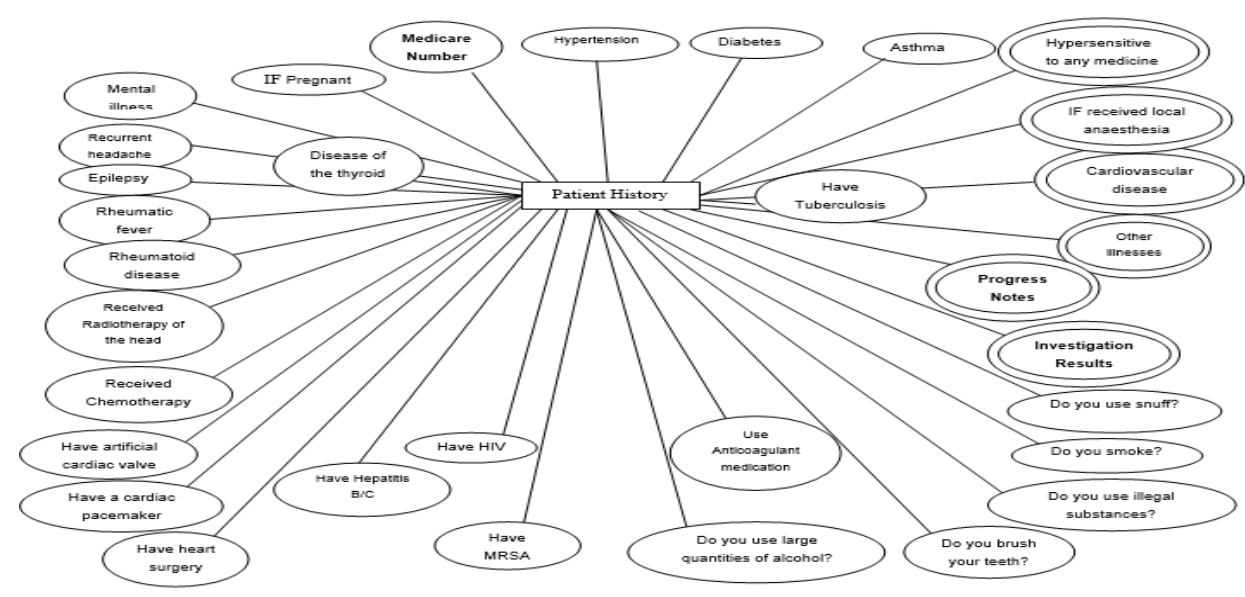

Fig 5: Patient History Structure

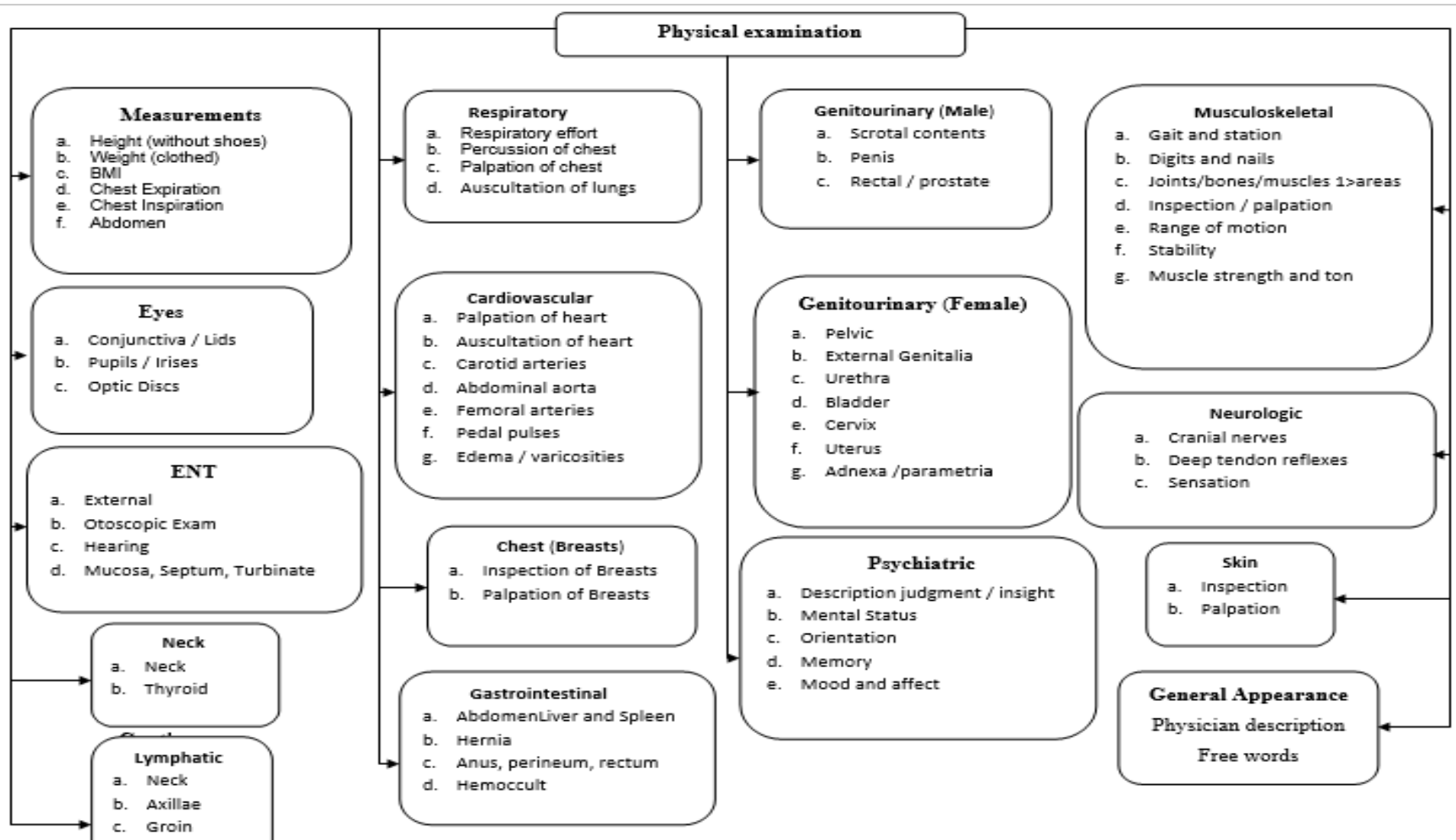

Fig 6: Patient Physical Structure 


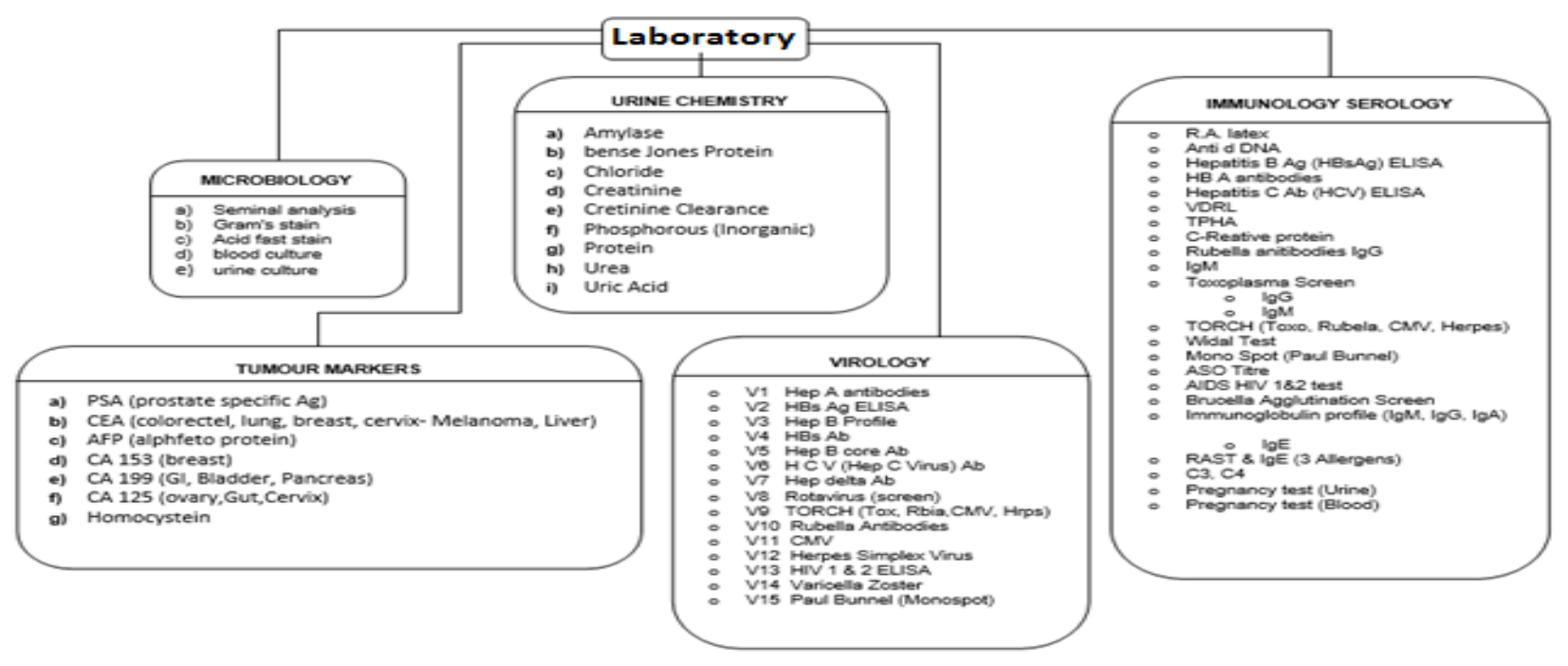

Fig 7: Patient Laboratory Results part 1

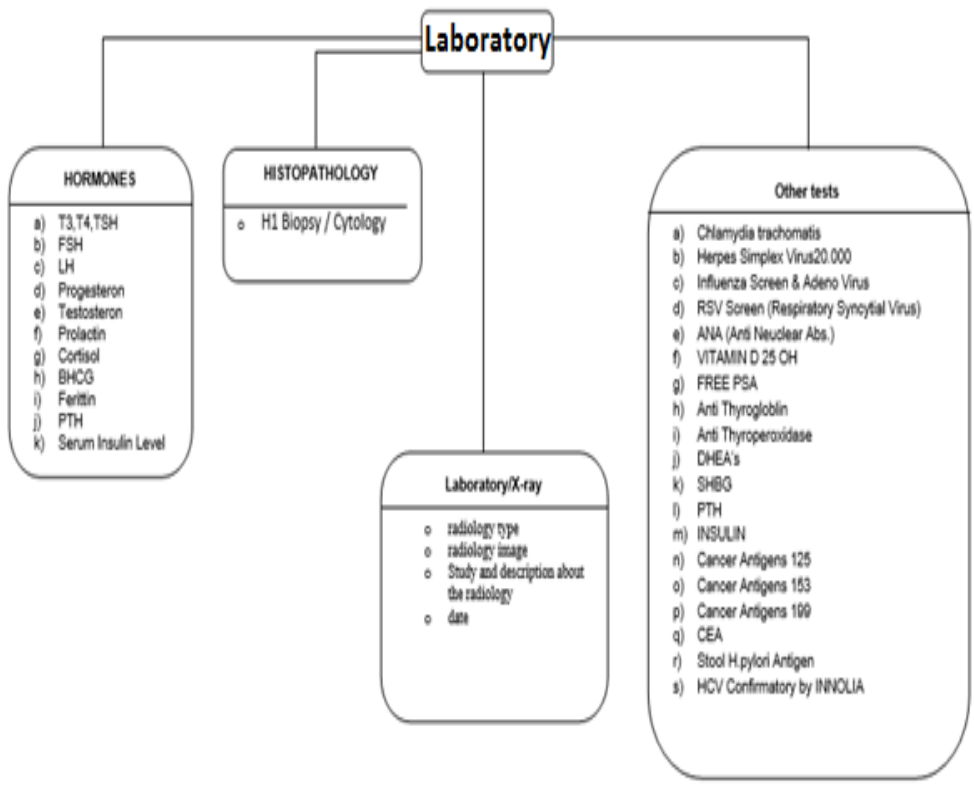

Fig 9: Patient Laboratory Results part 3

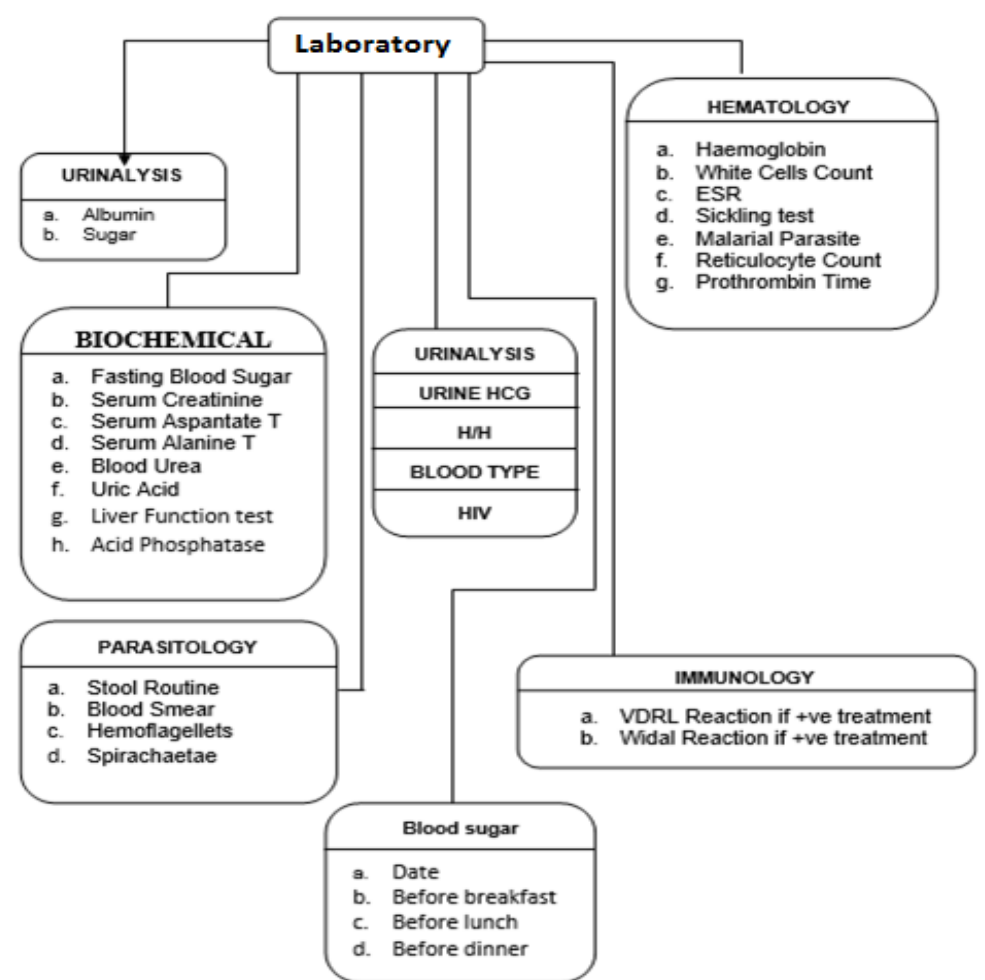

Fig 8: Patient Laboratory Results part 2

\section{Progress reports}

Progress reports are the part of a medical record where healthcare professionals record details to document a patient's clinical status or achievements during the course of a hospitalization or over the course of outpatient care.it serve as a record of events during a patient's care, allow clinicians to compare past status to current status, serve to communicate findings, opinions and plans between physicians and other members of the medical care team, and allow retrospective review of case details for a variety of interested parties. 


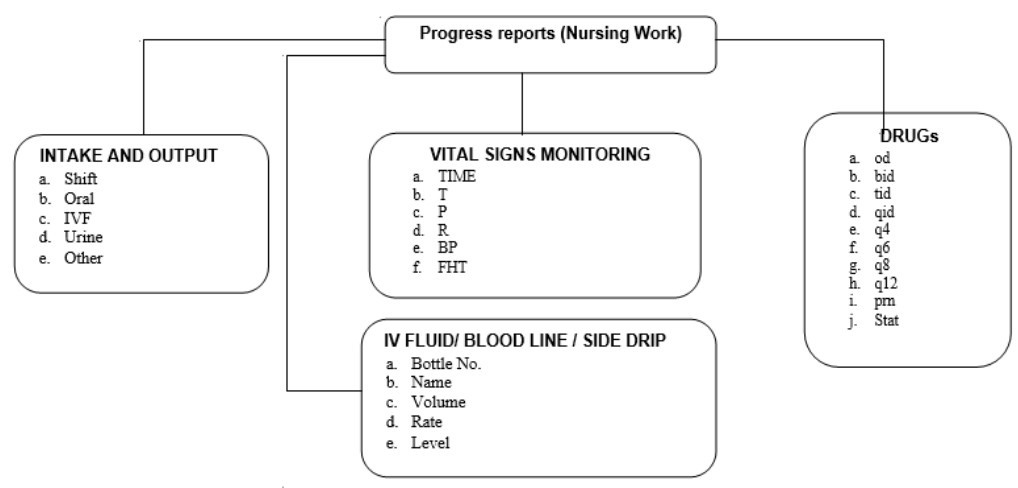

Fig 10: Progress Reports

\section{Allergy lists}

A drug allergy is an allergy to a drug, most commonly a medication. Medical attention should be sought immediately if an allergic reaction is suspected.

\section{Immunization status}

The process of inducing immunity to an infectious organism or agent in an individual or animal is through vaccination. Every country has its own Immunization schedule that should be stored in the EHR record.

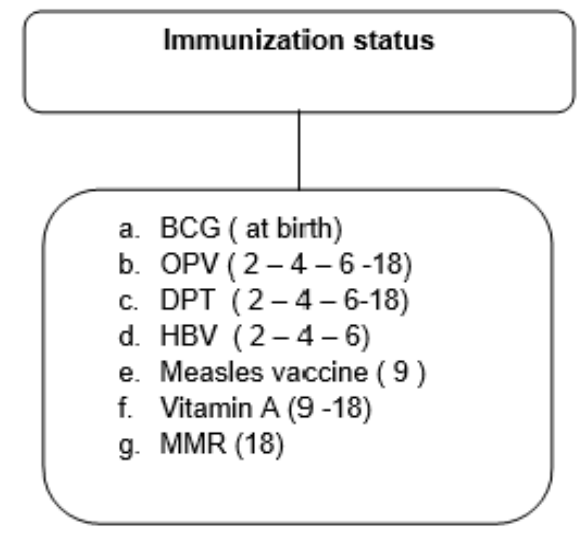

Fig 11: Patient Immunization status

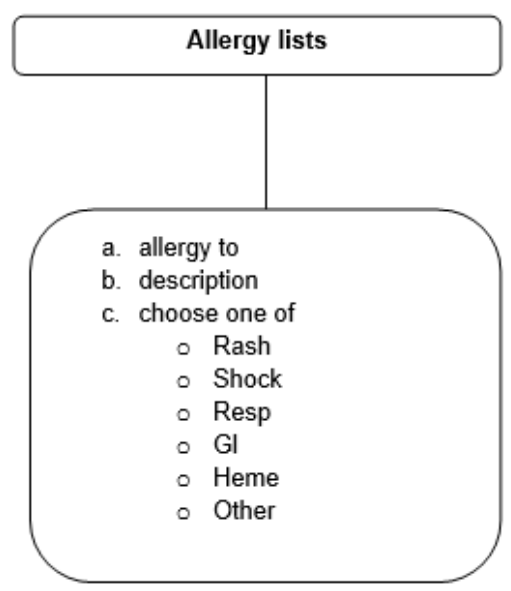

Fig12: Patient's Allergy lists

\section{Medication (Physician Investigation)}

Physician investigation represents the procedures that should be taken by patient after identifying his illness and diseases.

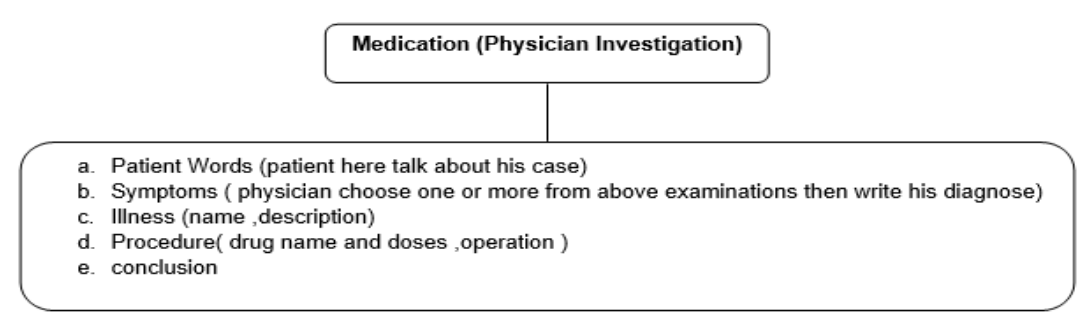

Fig 13: Medication 


\section{Appointment scheduling}

Appointment schedule is the part that is responsible for arrangement of patient visits to the clinics or hospitals.

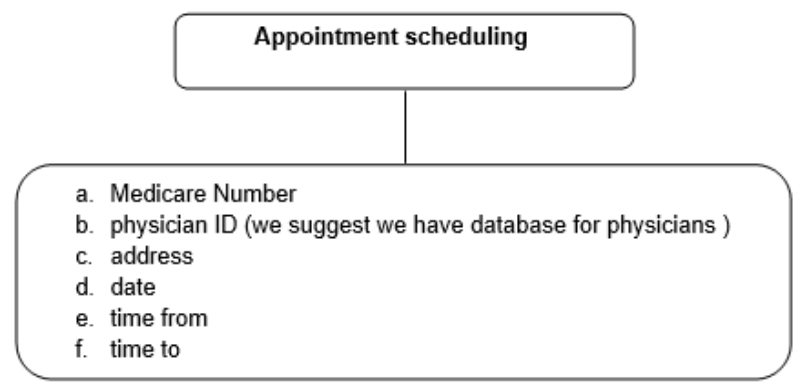

Fig 14: Appointment scheduling

\section{CONCLUSION}

The electronic health record became one of the important fields of study in the health information system area, for this reason several countries tried to implement their own health record systems. In this research we surveyed some of these attempts in Finland, France, Denmark and Egypt for identifying the strong and weak points in the implemented systems. This survey led to the lack of standard health record contents structure is the main barrier that face the implementation of the electronic health systems in these countries for this purpose we proposed a standard EHR content structure that succeeded in including all most important patient' medical data based on the international and national survey on clinical organizations. Applying this proposed structure leads to define a standard EHR contents that in turn make the patient electronic health record available on all clinical organizations that connected to the global EHR that achieve the operability of patient clinical data.

\section{References}

[1] Institute of Medicine. "Review of the Adoption and Implementation of Health IT Standards by the DHHS Office of the National Coordinator for Health Information Technology",http://www.iom.edu/,2006.

[2] Richard Dick, Elaine B. Steen, and Don Detmer, "The Computer Based Patient Record: An Essential Technology for Health Care", National Academy Press, 1997.

[3] HIMSS, Definition of an electronic health record. http://www.himss.org,2008.

[4] XianWen, Luo, and Wang Weiqing, "Improved Algorithms Research for Association Rule Based on Matrix" Intelligent Computing and Cognitive Informatics (ICICCI), the 2010 International Conference on IEEE, 2010.

[5] Miller, R. H. and Sim, Ida. "Physicians' Use Of Electronic Medical Records: Barriers And Solutions". Health Affairs, 2004.

[6] Waegemann, “EHR vs. CPR vs. EMR. Healthcare Informatics”, 2003.

[7] Himali Saitwala, Xuan Fengb, Muhammad Walji, Vimla Patel, Jiajie Zhanga, "Assessing performance of an Electronic Health Record (EHR) using Cognitive Task Analysis", Elsevierhealth, 2010.

[8] Lisa Pizziferri, Anne F. Kittler, Lynn A. Volk, Melissa M. Honourb, Sameer Gupta, Samuel Wang, Tiffany Wang, Margaret Lippincott, Qi Li and David W. Bates," Primary care physician time utilization before and after implementation of an electronic health record: A time-motion study", Elsevierhealth,2004.

[9] Working Group on Financial, Organizational, and Legal Sustainability of Health InformationConnecting for health,"Achieving electronic connectivity in healthcare", Markle Foundation, 2004.

[10] Lisa Pizziferri,Anne F. Kittler,Lynn.Volk, MHS,Lawrence N. Shulman,Jeffrey Kessler, Ginny Carlson,Taki Michaelidis,and David W. Bates," Impact of an Electronic Health Record on Oncologists' Clinic Time",AMIA,2005.

[11] Physician Micro System, Inc. A beginner's guide to internet-based healthcare applications”, McKesson, 2004.

[12] Vest, Joshua R., and Larry D. Gamm, "Health information exchange: persistent challenges and new strategies" Journal of the American Medical Informatics Association, Vol.17, No.3, 2010, pp. 288-294.

[13] E-health-Europe, http://www.ehealtheurope.net.

[14] Artmann J., Country Brief: France, ICT for Health Unit, 2010.

[15] Morten Bruun-Rasmussen, National IT strategies for the Danish Health Care service, Health Informatics Society, 2008.

[16] The Ministry of Communications and Information Technology, www.mcit.gov.eg 